\title{
The sedimentation of fine particles in liquid foams
}

\author{
Florence Rouyer, ${ }^{*}$ Christelle Fritz and Olivier Pitois
}

\author{
Received 25th February 2010, Accepted 26th April 2010 \\ DOI: $10.1039 / \mathrm{c} 0 \mathrm{sm} 00028 \mathrm{k}$
}

We investigate the sedimentation of fine particles in liquid channels of foams. The study combines numerical simulations with experiments performed in foams and in isolated vertical foam channels. Results show that particulate motion is controlled by the confinement parameter $(\lambda)$ and the mobility of the channel surfaces modelled by interfacial shear viscosity. Interestingly, whereas the position of the particle within the channel cross-section is expected to be a relevant parameter, it is shown that in foam there is no effect of this parameter on the sedimentation velocity. For low surface mobities, particles' velocities are small and almost independent on the size ratio; in opposition, for high surface mobilities, at low $\lambda$ the particle velocity is always larger than the Stokes velocity, whereas it is always smaller for $\lambda>0.5$. This behaviour has been understood from the generally observed position of moving particles along corners of the channels' cross-section.

\section{Introduction}

The transport of fine particles in aqueous foams is at the heart of the process of froth flotation for separating mineral ore from gangue. ${ }^{1}$ In broad outline, hydrophobic particles are attached to bubble interface and are recovered at the top of the flotation column, whereas hydrophilic particles remain in the liquid phase of the froth and are thus not expected to be collected. However, depending on their transport properties through liquid channels formed between the bubbles, hydrophilic particles are likely to be "captured" within the liquid phase of the rising froth. This is expected to occur as soon as the sedimentation velocity of those particles is smaller than the rising velocity of the froth, and this contributes to reduce the efficiency of the separation process. Through lack of a precise description for this transport, existing flotation models often consider that the sedimentation velocity of these particles is that of spheres in an unbounded liquid., Recently, the behaviour of particles within a quasi two-dimensional rising foam has been investigated. ${ }^{3}$ It was shown that for fine particles the foams channels (so-called Plateau borders-PB-) control the sedimentation velocity in opposition to nodes for large particles. Although interesting, the reported results do not help to establish a general law for particulate transport in Plateau borders. Based on a single foam channel experiment, Pitois et al. provided results for the settling behaviour of particles in settling motion along a vertical fluid channel with very mobile interfaces. ${ }^{4}$ It has been shown that for sufficiently small particles, slow and fast motions can be observed (the velocity corresponding to fast motions can be as high as twice the Stokes velocity in an unbounded fluid), depending on the particle position within the channel cross-section and the sphere/channel size ratio. Moreover, the fast motions are not observed anymore when the size ratio exceeds a critical value which has been found to be approximately equal to 0.5 . These experimental results were

Université Paris-Est, Laboratoire de Physique des Matériaux Divisés et des Interfaces, CNRS FRE 3300, 5 bvd Descartes, 77454 Marne la Vallée Cedex 2, France. E-mail: florence.rouyer@univ-mlv.fr; Fax: +3316095 72 97; Tel: +33160957263 modelled by numerical simulation with Stokes flows and slip condition (free boundary condition) at the walls of the channel. Note that, in such confined geometry, the particle can settle faster than in an unbounded liquid due to the presence of mobile interface, even so the inertia is negligible. Obviously, such results are of great interest for the modelling of particulate motions through the liquid channels of froth. However, a complete insight into this difficult problem should include the effect of mobility of the channel walls, which is known to depend on the surfactant used to stabilize the froth ${ }^{5,6,7}$ and currently modelled by the interfacial shear viscosity. In this paper, we focus on this aspect and we investigate the motions of fine particles along the Plateau borders of liquid foams. Both experimental and numerical results show that the mobility of the interface is precisely a central parameter in this problem.

\section{Experiments}

\subsection{Materials}

Two aqueous solutions are used: solution (A) TTAB $3 \mathrm{~g} \mathrm{~L}^{-1}$ (sodium dodecyl sulfate); and solution (B) TTAB $3 \mathrm{~g} \mathrm{~L}^{-1}+$ dodecanol $0.15 \mathrm{~g} \mathrm{~L} \mathrm{~L}^{-1}$. For both solutions, the surfactant concentration is three times greater than the critical micelles concentration, we thus assume that the surfactant concentration at the interface is always at equilibrium, considering that adsorption time of surfactants at the interface is short enough compared to the settling time at the scale of the particle. Consequently we neglect Marangoni stress compared to surface shear stress. Solution (A) is well known to produce "mobile" liquid-gas interfaces, corresponding to extremely low interfacial shear viscosities $\left(\mu_{s}\right): 10^{-5} \mathrm{~g} \mathrm{~s}^{-1}{ }^{7}$ Addition of dodecanol to solution (A) is known to induce a significant increase of the surface viscosity ${ }^{7}$ and greater than tenfold the surface viscosity of solution (A), so that the interfaces obtained from solution (B) will be referred to as "non-mobile". The bulk shear viscosity $\mu$ and density of solutions are equal to those of pure water. Surface tensions are $\sigma=37 \mathrm{mN} \mathrm{m}^{-1}$ for solution (A) and $\sigma=27 \mathrm{mN} \mathrm{m}^{-1}$ for solution (B). 
Solid particles are silica spheres (Duke Scientific Corporation, density $\rho_{\mathrm{S}}=2450-2500 \mathrm{~kg} \mathrm{~m}^{-3}$ ). Diameters $\mathrm{d}$ are in the range 15$140 \mu \mathrm{m}(\Delta \mathrm{d} / d \approx 5 \%)$. The maximal particulate Reynolds number $\left(R e_{p}\right)$ is equal to 3.4 for the largest particles and less than or equal to 0.1 for particle diameter smaller than $45 \mu \mathrm{m}$. A dedicated delivery system was developed to introduce the particles into the PB channels without additional liquid flow. The particles are first mixed with the foaming solution and placed in a modified 3-path valve, ensuring their transfer to the feed tube where they are allowed to settle.

\subsection{Foam experiment}

The experimental cell consists of two cylindrical glass containers separated by a sintered glass disk (Fig. 1). The cell is first filled with the foaming solution and bubbles with $2.5 \mathrm{~mm}$ diameter are generated in the upper container (diameter: $50 \mathrm{~mm}$, height: 100 $\mathrm{mm}$ ) by blowing filtered nitrogen through a capillary. A lid is used to ensure water vapour saturation inside the foam cell. The lower container is connected to a liquid reservoir. Decreasingrespectively increasing - the vertical position (L) of the liquid reservoir with reference to the cell enables us to decreaserespectively increase - the liquid pressure inside the cell. As a result, liquid solution is forced out of - respectively into - the foam from the bottom and the Plateau border cross-sections increase - respectively decrease - as long as the foam remains in contact with the base of the container, ${ }^{8}$ this was achieved by controlling the hydrophobicity of the inner cell wall as well as of the porous plate (a solution of perfluorodecyltrichlorosilane was used for coating). Under these conditions, the radius of curvature of PBs located in the plane at height $\mathrm{z}$ inside the foam is given by $R_{P B}(z) \approx \frac{\sigma}{\rho g(z-L)}$ and is typically in the range $100-200 \mu \mathrm{m}$.

A 3D micropositioner is used to drive the delivery tube to the desired position inside the PB network. Images of PBs inside the foam (a few bubbles far from the cell wall) can be grabbed using a CCD camera set on a 3D translation stage and connected to a computer. Magnification levels allowed images of one or several PBs (including nodes) to be obtained. The camera and the cell were oriented in such a way that the studied PBs were located in a plane perpendicular to the optical axis of the camera. As an illustration of the particle tracking procedure, Fig. 2 presents an

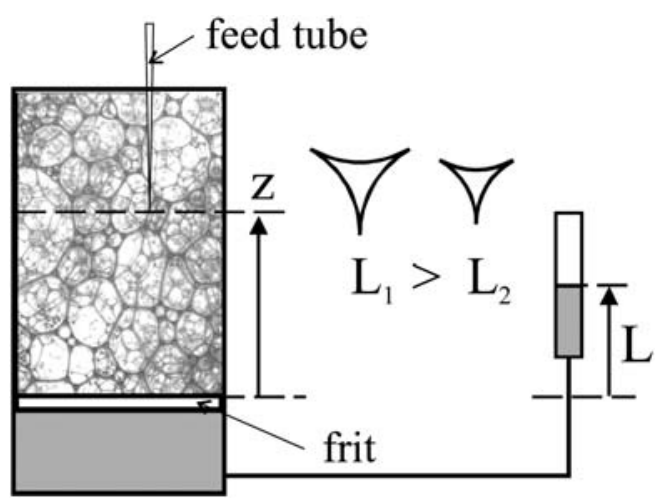

Fig. 1 Sketch of the foam cell connected at the bottom to a liquid reservoir which controlled altitude permits to vary the size of the channel cross section.

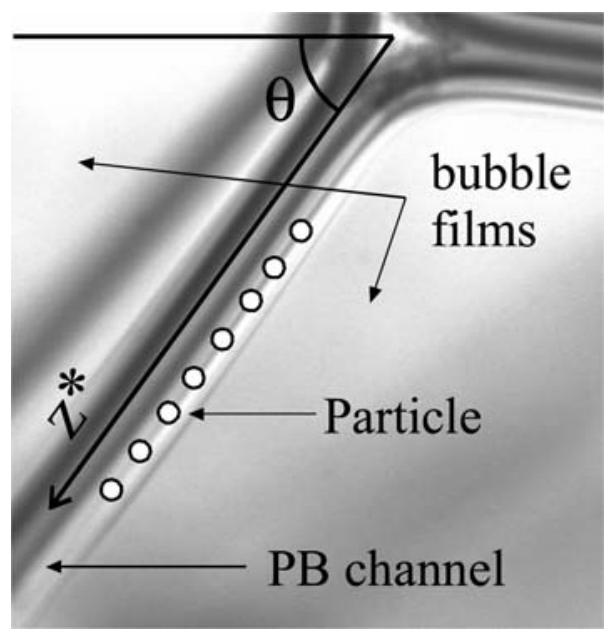

Fig. 2 Successive positions occupied by a glass sphere $(35 \mu \mathrm{m})$ in settling motion through a Plateau border in a foam of solution B, the acquisition time is $\Delta \mathrm{t}=200 \mathrm{~ms}$.

example of the successive positions occupied by a sphere in sedimentation through investigated foams.

\subsection{Single vertical channel experiment}

This experiment has been shown to be useful for the study of particulate motions in Plateau borders, especially for the study of the position of the sphere within the channel cross-section. It is described in details in ref. 4 and we briefly recall that the vertical channel is obtained by withdrawing a dedicated frame from a reservoir containing the foaming solution. The frame consists of a vertical metallic tube on which three rods (diameter $1 \mathrm{~mm}$ ) are fixed. Due to capillary forces, the cross-section of the resulting vertical liquid channel (Plateau border) is almost an equilateral triangle except that the three segments joining the corners are tangent circular arcs of radius $R_{P B}$. Each corner joins to a thin vertical liquid film - of negligible thickness - attached to the holder and stabilized with surfactants contained in the solution. The frame is accurately positioned with reference to the reservoir to easily adjust the length of the channel (typical lengths are in the range $5-10 \mathrm{~mm}$ ).

The inner diameter of the holder is $10 \mathrm{~mm}$ and a circular outlet of diameter $1 \mathrm{~mm}$ is opened at its lower part: it is used to deliver the particles through the channel. Images of the channel during the experiments were grabbed through windows in the cell cover. The successive positions occupied by a sphere along the channel axis are then determined using simple image processing procedures. Another window was also placed at the bottom of the liquid reservoir. In illuminating the channel from the top (through the holder) and using a high speed camera equipped with an appropriate lens, pictures of the channel cross-section could be obtained during particles motion. The pictures are then used to determine the position of the particle within the channel cross-section during the sedimentation and to estimate de radius of curvature of the Plateau border: $R_{P B} \approx 400 \mu \mathrm{m}$. As this experiment is dedicated to the study of the position of the sphere within the channel cross-section, we focussed on the smallest 
sphere/channel size ratio, for which the number of different positions that can be distinguished experimentally is the largest.

\section{Numerical simulation}

To model the settling velocity $\mathrm{V}$ of a particle along a Plateau border made of a fluid interface we solve the fluid flow by use of COMSOL Multiphysics software. The Plateau border geometry is delimited by 3 tangential cylinders of radius $R_{P B}$ and length $4 R_{P B}$, this latter has been chosen large enough such that it does not influence results (see Fig. 3a). The position of the particle is set at the middle height of the channel and is varied along the median $x$ axis (see Fig. 3b). The origin of $x$ is chosen in the middle of the section and the $x$ axis is oriented positively in direction of a corner. We note that the three corners of the Plateau border cross-section are equidistant (equilateral triangle with length $R_{P B}$ ) and the distance between one corner to the center is equal to $R_{P B} / \sqrt{3}$. The parameter $\lambda=d / d_{\text {lim }}$ is the confinement parameter; it compares the size of the particle with the maximum diameter of the circle inscribed in the PB cross-section: $\quad d_{\lim }=2\left(2 / 3 R_{P B} \sqrt{3}-R_{P B}\right)=2 R_{P B}(2 / \sqrt{3}-1)$. Four values of $\lambda$ have been studied systematically: 0.2 , $0.4,0.6$ and 0.8 . For each $\lambda$, the position of the particle is within two extremes values $0.97 x_{\min }$ and $0.97 x_{\max }$ that verifies: $\quad x_{\min }=d / 2-d_{\lim }=-\frac{2 R_{P B}}{\sqrt{3}}+R_{P B}+d / 2$ and $x_{\max }=\frac{R_{P B}}{\sqrt{3}}-\sqrt{\left(R_{P B}+\frac{d}{2}\right)^{2}-R_{P B}{ }^{2}}=\frac{R_{P B}}{\sqrt{3}}-\sqrt{R_{P B} d+\frac{d^{2}}{4}}$

(see Fig. 3b).

We use an "auto" extra-fine mesh refined near the particle (maximum element size $=0.02$ and growth rate $=1.1$ ) and the

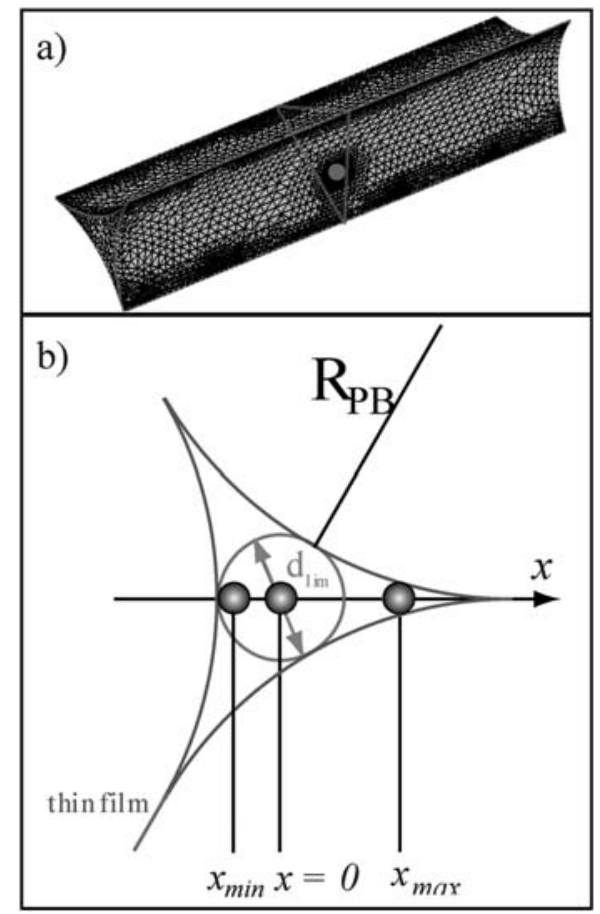

Fig. 3 (a) Example of mesh used to compute the settling velocity of a sphere inside a Plateau border. The sphere (for $\lambda=0.4$ ) drawn in red colour. (b) Sketch of a Plateau border cross-section. corner of the PB (maximum element size $=0.05$ and growth rate $=1.05$ ) as illustrated in Fig. 3. For the smaller confinement parameter $(\lambda=0.1)$, we refined even more the mesh size close to the particle.

We consider dimensionless Stokes equations (fluid density and viscosity are respectively set to zero and one in "the Fluid Dynamic, Incompressible Navier-Stokes" user interface). The simulations are run in the frame of the particle and zero velocity is imposed at the surface of the particle (no-slip conditions). Fluid velocities at the entrance and the outlet of the channel, as well as the one of the edges of the Plateau border, are constrained to the parameter $-V_{p}^{*}$. The dimensionless viscous force $F_{V}^{*}$ exerted on the sphere is calculated from the integral of the Lagrange multiplier along the axis of the channel. The fluidity of the interface is modelled using weak terms ${ }^{9}$ for balancing the bulk viscous stress with the surface viscous stress on the circular boundary $\mu \mathbf{n} . \nabla v=\mu_{s} \Delta_{s} v$ where $v$ is the velocity of the fluid. The two relevant parameters are the Boussinesq number $B o$ and $B o^{\prime}$ which compare shear stress of the interface to the shear stress of the bulk at the scale of the channel: $B o=\mu_{s} / \mu R_{P B}{ }^{10}$ and at the scale of the particle: $B o^{\prime}=\frac{\mu_{s}}{\mu d}=\frac{B o}{2 \lambda(2 / \sqrt{3}-1)}{ }^{11}$ The parameter $B o$ is varied from 0.001 to 1 . For given $\lambda, x$ and $B o$, a parametric simulation that varied $V_{p}^{*}$ is run for at least three values of $V_{p}^{*}$ that permit to check for the proportionality relation: $F_{V}^{*}=\alpha V_{p}^{*}$. Indeed, we are concerned with low particulate Reynolds number, so that the only hydrodynamic force is the viscous drag $F_{V}=f 3 \pi d V_{p}$ where $f$ is a correction factor due to the presence of the channel walls. Balancing $F_{V}^{*}$ with the dimensionless buoyancy force of the sphere $\left(F_{g}^{*}=3 \pi d\right)$, we thus deduce the normalized terminal settling velocity of the particle for: $V_{p}^{*}=v / v_{\text {Stokes }}=1 / f=$ $3 \pi d / \alpha$.

\section{Results}

A typical example of the measurements performed with the foam experiment is presented in Fig. 4. In this figure, $\Delta \mathrm{z}^{*}=\mathrm{z}^{*}-\mathrm{zo}^{*}$, where $\mathrm{zo}^{*}$ is the position $\mathrm{z}^{*}$ for which the particle reach a stable position in the cross-section of the channel. It is important to note that as the particles enter a PB channel (from a node), it needs some distance for them to find a stable position in the channel cross-section, over which the velocity evolves toward a constant value. From the present results, it appears that the

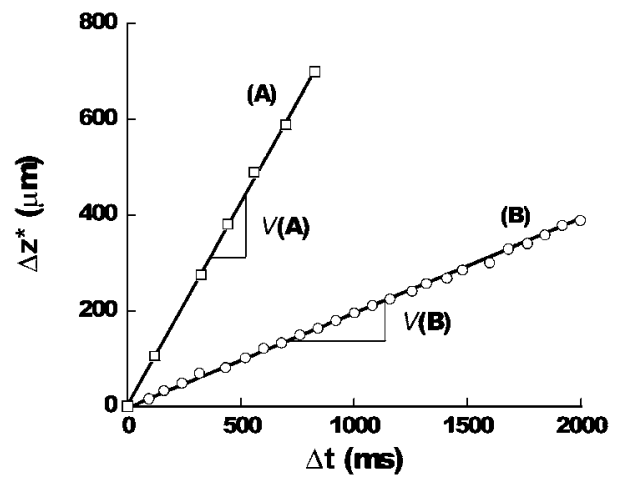

Fig. 4 Typical example of the position measurements as a function of time performed with the foam experiment for solution A (squares) and solution B (circles). 
particles reach a constant velocity $(V)$ with the two foaming solutions. As a qualitative information, it is deduce from Fig. 4 that $V(\mathrm{~A})>V(\mathrm{~B})$, as expected from the lower "mobility" of solution (B).

Velocities measured for particles motions in the foam are presented in Fig. 5. We have grouped results obtained for Plateau borders inclined of angle $\theta=55 \pm 2^{\circ}$ and $\theta=70 \pm 2^{\circ}$, for which data were significant; the effect of this angle is taken into account in the reported values: $V_{p}^{*}=V /\left(V_{\text {Stokes }} \sin \theta\right)=1 / f$. This set of data confirms the information given by Fig. 4: sedimentation velocities are strongly dependent on the surfactant used to stabilize the interfaces. Indeed, two distinct curves appear in spite of some scatter in the data, each curve corresponding to a given solution. This "mobility" effect is very large for low values of $\lambda$, but is strongly reduced as $\lambda$ increases, although always noticeable. More quantitatively, at low $\lambda$ the measured velocity for solution (A) is twice the Stokes velocity, which is tenfold the one measured for solution (B). For this latter, all measured velocities are smaller than the Stokes velocity. This shows that the interfacial mobility is a central parameter in this problem. The confinement parameter $\lambda$ has a great influence on the motions of spheres in foam (A), but there is no evidence for such an effect for solution (B).

Experimental velocity values obtained for solution (B) are reported as a function of the position of the particle along the median axis in Fig. 6, and compared with results already published for solution (A). ${ }^{4}$ As already explained, we focussed on the smallest confinement parameter, i.e. $\lambda=0.2$, for which the number of positions that can be distinguished experimentally is the largest. The velocity appears to be: minimum as the sphere locates at the corner of the channel cross-section, maximum as it locates in the central area of the cross-section. It can be said that the velocity decreases as the sphere settles closer to the walls. A normalized velocity $V_{p}^{*}=V_{2} \approx 0.2$ is measured as the sphere is

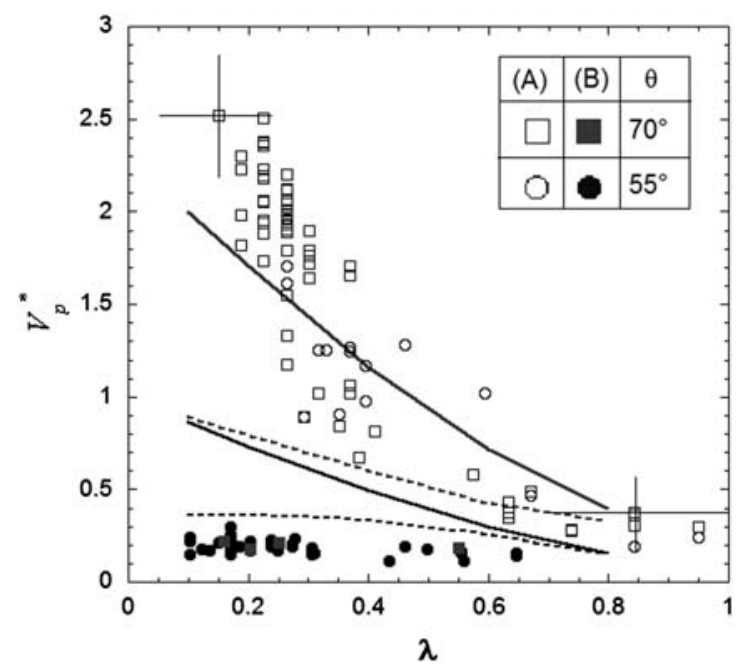

Fig. 5 Normalized settling velocity as a function of confinement parameter: Experimental data measured within foam made from solutions (A) and (B) are respectively represented by open and full symbols. Minimal (dash line) and maximal (continuous line) values obtained from numerical simulations for $B o=0.001$ and 1 are respectively presented by grey and black lines.

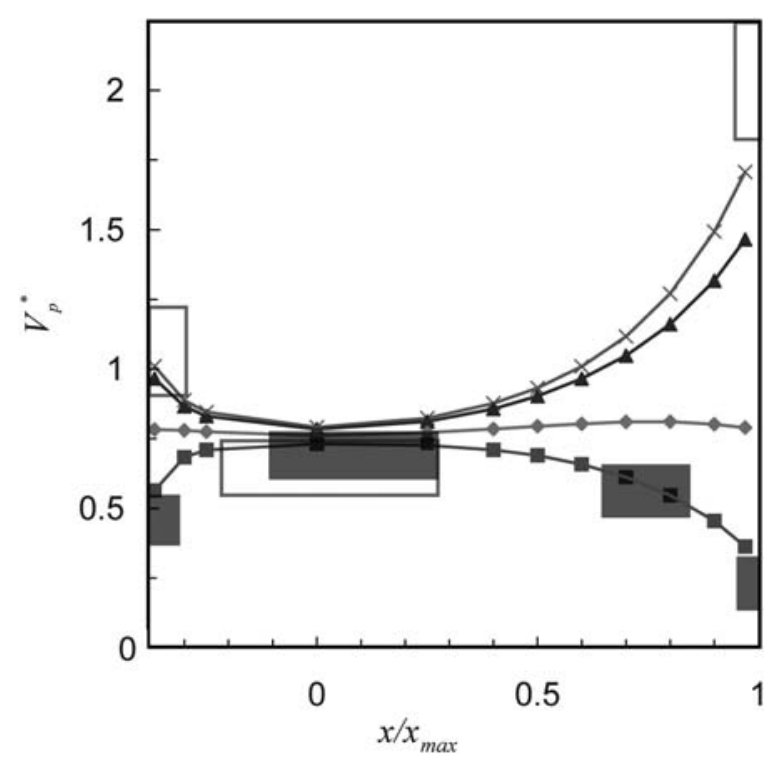

Fig. 6 Normalized settling velocity as a function of $\mathrm{x}$ for $\lambda=0.2$. Experimental data obtained in the Plateau border apparatus for solution (A) and (B) are respectively represented by open and grey boxes. Numerical results are represented by squares, diamonds, triangles and times lines for respectively for $B o=1,0.1,0.01,0.001$.

close to two walls (corner), $V_{p}^{*}=V_{1} \approx 0.4$ as it is close to one wall, $V_{p}^{*}=V_{0} \approx 0.7$ as there is no wall close to the sphere. The normalized velocity $V_{0}$ for spheres in the central zone measured for solution A and solution B are identical.

Dependence of the numerical velocity with the sphere position is presented in Fig. 6 for $B o=0.001,0.01,0.1$ and 1. For small $B o$ values, the velocity is maximum as the sphere is at the corner of the cross-section, minimum as it is at the centre of the crosssection, and intermediate as it is at the opposite interface. For the larger $B o$ value, this order reverses: the velocity is minimum as the sphere is at the corner of the cross-section and maximum as it is at the centre of the cross-section. For the intermediate value of $B o$, the dependence of the velocity with the sphere position is very weak and the profile is essentially flat. For position of sphere in the central zone, the influence of $B o$ is weak.

In Fig. 5, numerical values for maximum and minimum sphere velocities are plotted as a function of $\lambda$ for $B o=0.001$ and $B o=$ 1. It can be noted that: (i) velocities corresponding to $B o=0.001$ are larger than those for $B o=1$; (ii) velocities are decreasing functions of $\lambda$; (iii) for both $B o$ values, the $\lambda$-dependence is stronger for the maximum velocity than for the minimum velocity.

\section{Discussion}

Results for the sedimentation of fine particles in foams (A) and (B) have revealed a very strong effect of the interfacial mobility, especially at low $\lambda$ (Fig. 5). Comparisons with data obtained from both the vertical Plateau border experiment and numerical simulations of Fig. 6 suggest that the measured velocity in foam is always close to the velocity expected at a corner of the channels cross-section, i.e. (i) maximum for high interfacial mobility (solution A, small Bo), (ii) minimum for low interfacial mobility 
(solution B, large Bo). During the foam experiment, the position of the sphere is not measurable with precision. However, we generally observed that the axis of the sedimentation motion in foam channels is often close to a corner. This is because in foams, the channels are inclined with respect to the vertical axis, so that the gravity force always acts to position the particle at the lowest stable position in the channel cross-section, i.e. a corner. Even if the particle were positioned along the central axis of a perfectly vertical channel (a situation that we never observed during foam experiments), it would approach the wall in the following channel. Note that other situations, where the sphere moves along the opposite interface for example, are nevertheless possible but much less probable.

Now we discuss quantitatively the velocity values obtained for channels with low interfacial mobility (solution B). Fig. 5 clearly shows that $V_{p}^{*}$ is only weakly dependent on the parameter $\lambda$. More precisely, no evident dependence is observed for values of $\lambda$ smaller than $\approx 0.3$. As already explained, these velocities are representative of the velocity of spheres in settling motion along a corner of Plateau border channels, referred to as $V_{2}$, and correspond to the minimum velocity for each value of $\lambda$. In that case, the particle slow down is mainly due to the shearing of the liquid layer between the sphere and the closest wall, ${ }^{12}$ rather than due to the back flow of the fluid through the gap between the sphere and the confining walls. ${ }^{13}$ This is in contrast with the settling of spheres axially centred in vertical tubes, ${ }^{14,15}$ which is often used as a reference to model the motion of particles confined by solid walls, and for which a significant dependence of the drag coefficient with $\lambda$ is observed. For $\lambda=0.2$, both experiments provide a value for $V_{2}$ close to 0.2 . This suggests that the normalized velocity is not influenced by the inclination angle in the range of investigated values $\left(50^{\circ}-90^{\circ}\right)$. The numerical value obtained for $B o=1$ and $x=0.97 x_{\text {max }}\left(V_{2}=0.3\right)$ is also in good agreement (considering that a better agreement would be obtained if the minimum gap imposed in the simulation was reduced to zero). Extrapolating the data for $B o=1$ in Fig. 6 to $x=x_{\max }$, one can estimate $V_{2}$ $\approx 0.2$, suggesting that mobility parameter of solution (B) has been set to an appropriate value in the simulation. The corresponding value for the surface shear viscosity is approximately equal to $4.10^{-4} \mathrm{~g} \mathrm{~s}^{-1}$. It is in agreement with generally reported value for this solution. ${ }^{7}$ Note that the numerical model does not consider the rolling of the sphere. However, rolling was reported for spheres in settling motions along the bottom wall of inclined solid channels. ${ }^{16,17}$ Although the wall of the Plateau border channels is fluid, both slipping and rolling motions were distinguished during experiments with solution B (but we were not able to measure accurately the rotation with the present tracking method). For inclined solid circular tubes (infinite $\mathrm{Bo}$ ), the drag coefficient has been reported to be almost independent on $\lambda$ if $\lambda<0.4$ and if the Reynolds number is smaller than unity $^{16}$ (note within our experimental conditions, the Reynolds number of the particle satisfies the condition: $R e_{p}<0.1$ for $\lambda<$ $0.4)$. In that case, the drag coefficient can be expressed by the simple relationship: $C_{D} \approx 225 / R e$, so that $V_{p}^{*} \approx 0.1$. This value is smaller than the one reported in this study $(0.2)$ and this deviation reflects the difference in the geometrical configuration of the "contact" as well as the finite value of the surface shear viscosity.
The case of highly mobile interfaces (solution A) is discussed now. In stark contrast with solution $\mathrm{B}$, the experimental curve (Fig. 5) now exhibits a strong dependence of $V_{p}^{*}$ on the parameter $\lambda$. As might be expected, due to the high mobility of the interface, the values of the drag coefficient are smaller than those obtained for solution B. Similarly to the maximal sphere velocity in a vertical $\mathrm{PB}$; two distinct regimes are observed: for high values of $\lambda(\lambda>0.3-0.5)$ the sphere moves slower with respect to the unbounded case (slow motion regime), whereas for low values of $\lambda(\lambda<0.3-0.5)$ the sphere moves faster with respect to the unbounded case (fast motion regime). In the latter regime, the sphere velocity can be as high as twice the value of the velocity in the corresponding unbounded case, in spite of the confining effect of the channel walls. It is to noted that no rolling of the sphere was observed with solution A. This large value for $V_{2}$ is obtained from both experiments and from the numerical simulation with $B o=0.001$. The corresponding surface viscosity is $4.10^{-7} \mathrm{~g} \mathrm{~s}^{-1}$, which is quite lower than values reported for this solution. Note also that numerical results compare quiet well with experimental data for every $\lambda$, and that the critical value corresponding to the transition between slow motion and fast motion regimes is found to be equal to $\approx 0.5$. This behaviour is very similar to results for infinitely mobile interface (free boundary condition), meaning that below a certain value, decreasing the surface viscosity of the interface has insignificant effect.
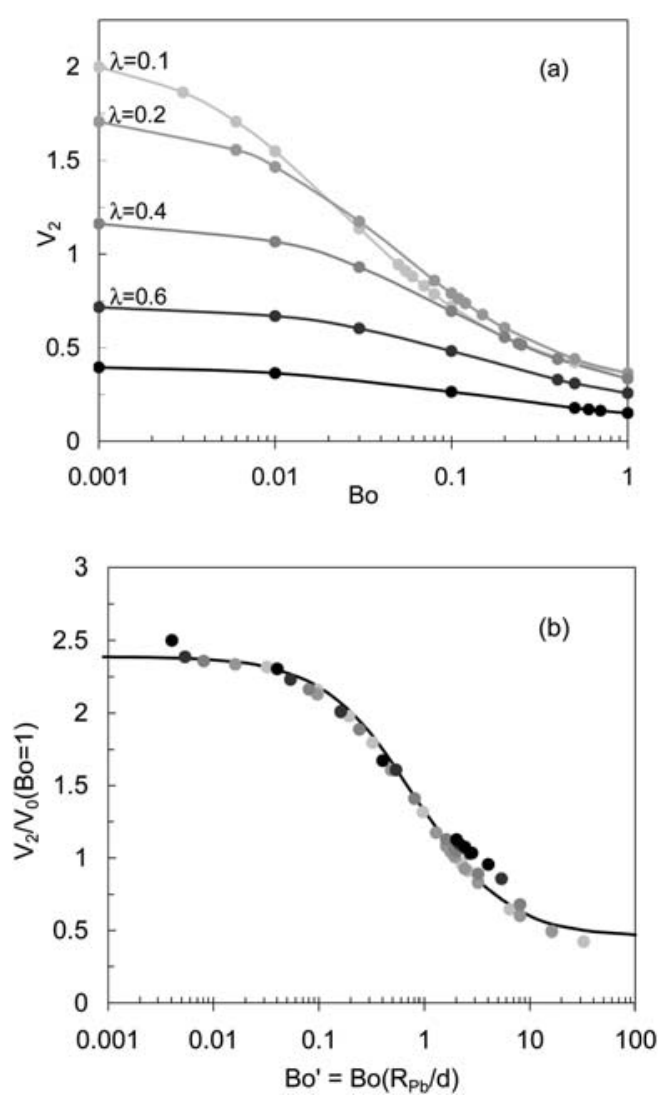

Fig. 7 Numerical results of the normalized settling velocity of sphere in a corner of a Plateau border $V_{2}$ for different surface mobility and for confinement parameter varied from 0.1 to 0.8 . (a) $V_{2} v s$. $B o$; (b) $V_{2}$ is normalized by $V_{0}$ for $B o=1 \mathrm{vs}$. $B o^{\prime}=B o R_{P B} / \mathrm{d}$. 
As $V_{2}$ is the relevant velocity for sedimentation of fine particles in foams, it is of interest to precise the complete dependence of this velocity with both confinement and mobility parameters. To this respect, Fig. 7a presents a complete set of numerical data for $V_{2}$ (we recall that due to numerical limitations this values are obtained for $x=0.97 x_{\max }$ ). Qualitatively, the main features of this behaviour are: (i) at low $\lambda, V_{2}$ is strongly decreased as $B o$ increases, (ii) at low $B o, V_{2}$ is strongly decreased as $\lambda$ increases, (iii) at high $\lambda$ or high $B o, V_{2}$ remains close to $\approx 0.3$. Note that for $B o$ larger than approximately 0.01 , numerical results indicate that $V_{2}$ is smaller for particles with $\lambda=0.1$ than for particles with $\lambda=0.2$. This result can be understood in considering that for such small values of $\lambda$, the effect of the backflow is negligeable (as the sphere is at the corner). In other words, the precise shape of the channel far from the sphere has not a significant influence on the sphere velocity; this latter is essentially controlled by the geometry and the mobility of the closest walls. As a consequence, the relevant length scale accounting for interfacial shear is the size of the sphere (instead of the size of the channel) and the relevant mobility parameter is $B o^{\prime}$. This is similar to the settling motion of a particle between two vertical viscous interfaces, for which the effect of the backflow is negligible and $1 / f$ decreases with $B o^{\prime} .{ }^{11}$ Consequently, for a given $B o$, i.e. a given $R_{P B}$ and a given $\mu_{s}$ in our case, $B o^{\prime}$ decreases with the particle size, and $1 / f$ increases with $\lambda$, for small $\lambda$ and negligible backflow effects. Indeed, the increase of $\lambda$ induced a slow down effect due to the backflow which superimposes to the effect of mobility. It is tempting to model the global behaviour in considering independently the two basic effects. This suggests to write $V_{2}$ as: $V_{2}(\lambda, B o)=g\left(B o^{\prime}\right) h(\lambda)$, where $g\left(B o^{\prime}\right)$ accounts for the mobility effect and $h(\lambda)$ accounts for the backflow effect. We argue that this latter can be reasonably assessed by the evolution for the velocity of spheres axially centred in the channel, i.e. $V_{0}$. This is supported by the fact that $V_{0}$ is only weakly dependent on $B o$ over the range of three decades investigated in this study (see Fig. 5 for example). For the estimation of the confinement effect, we choose $h(\lambda)$ such that $h(\lambda) \cong V_{0}(\lambda, B o=1)$. The confinement factor is classically described using polynomial functions, ${ }^{15}$ so that we approximate $h(\lambda)$ by $h(\lambda)=1-1.488 \lambda+0.535 \lambda^{2}$ (the deviation with numerical data is less than $1 \%$ for $\lambda<0.6$ ). In Fig. 7b we plot $V_{2} / h(\lambda)$ as a function of $B o^{\prime}$, showing that numerical data approximately collapse on the same curve. This supports our simple approach and allows for the function $g\left(B o^{\prime}\right)$ to be determined: $g\left(B o^{\prime}\right)=\left(4.3+B o^{\prime}\right) /\left(1.8+2.2 B o^{\prime}\right)$, thus providing a useful expression to estimate the velocity of particles within the channel of foams.

Finally, we discuss the deviation between the maximum and the minimum velocities. As the interfacial shear viscosity increases from low to high values, $V_{2}$ evolves from $V_{2}>1$ (the maximum velocity) to $V_{2}<1$ (the minimum velocity), whereas in the same time, $V_{0}$ remains almost unchanged. As a consequence, there exists a particular value of $B o$ for which the deviation between the maximum and the minimum velocities becomes very small or even vanish. In Fig. 8, we plot $\Delta V=\left(\max \left(V_{0}, V_{1}, V_{2}\right)-\right.$ $\left.\min \left(V_{0}, V_{1}, V_{2}\right)\right)$ as a function of $B o$. For every $\lambda$ value, a particular value of $B o$ is found for which $\Delta V \approx 0$, this value correspond to $B o^{\prime}=2$. Note that $B o^{\prime} \approx 2, V_{2} \approx V_{0}(\lambda, B o=1)$. In that particular case, the velocities of spheres in settling motions along a corner or along the central axis of the channel are almost the

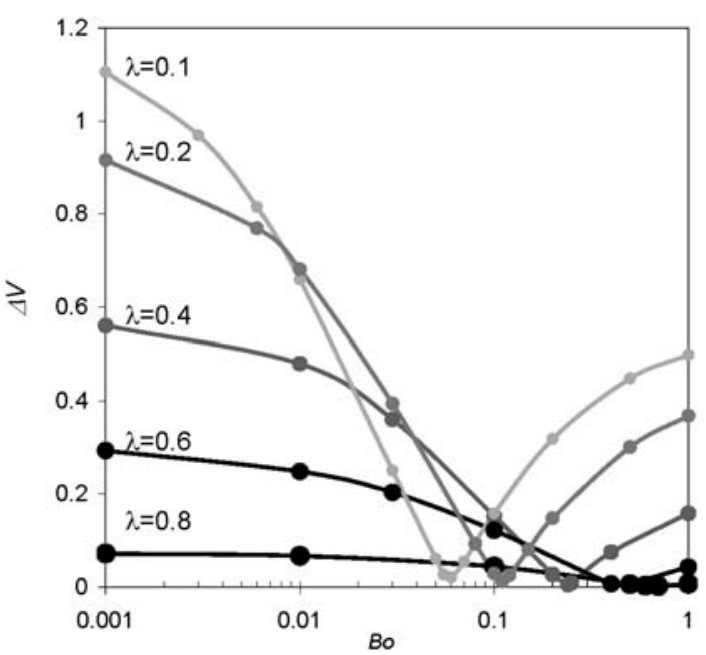

Fig. 8 Numerical results of the amplitude of the deviation $\Delta V$ as a function of $B o$ for confinement parameter varied from 0.1 to 0.8 .

same. This is illustrated in Fig. 6 for $B o=0.1$ and $\lambda=0.2$, where the velocity profile is almost flat over the channel cross-section.

\section{Conclusion}

We investigated the gravity motion of fine particles in liquid channels of foams. The study combined numerical simulations with experiments performed both in foams and in isolated vertical foam channels. For vertical channels, results show that the motion is controlled by the particle/channel size ratio $(\lambda)$, the mobility of the channel surfaces (Bo), as well as the position of the particle within the channel cross-section. For small $B o$ values, the velocity is maximum as the sphere is at the corner of the cross-section, minimum as it is at the centre of the cross-section, and intermediate as it is at the opposite interface. For the larger $B o$ value, this order reverses: the velocity is minimum as the sphere is at the corner of the cross-section and maximum as it is at the centre of the cross-section. We showed that for every $\lambda$, there exists a value of $B o$ for which the deviation between the maximum and the minimum velocities reduces to a vanishing small value: in that particular case, the dependence of the velocity with the sphere position is very weak. Interestingly, in foam, there is no effect of the position parameter on the sedimentation velocity. For low surface mobility, particles velocities are small and almost independent on the size ratio; for high surface mobility, we found that at low $\lambda$ the particle velocity is always larger than the Stokes velocity, whereas it is always smaller for $\lambda$ $>0.5$. This behaviour has been understood from the generally observed position of settling particles along corners of the channels cross-section.

\section{Notation}

$B o=\mu_{s} / \mu R_{P B} \quad$ Boussinesq number defined at the scale of the

$B o^{\prime}=\frac{\mu_{s}}{\mu d}$

$d \quad$ particle diameter

$d_{\mathrm{lim}} \quad$ maximum diameter of the circle inscribed in the PB cross-section 


\begin{tabular}{|c|c|}
\hline & $\begin{array}{l}\text { correction factor for the viscous drag due to } \\
\text { the presence of the channel walls }\end{array}$ \\
\hline$\lambda=d / d_{\lim }$ & confinement parameter \\
\hline$R e_{p}$ & particulate Reynolds number \\
\hline$R_{P B}$ & Plateau border radius of curvature \\
\hline$V$ & settling velocity of the particle \\
\hline$V_{p}^{*}$ & $\begin{array}{l}\text { settling velocity normalized by Stokes velocity } \\
=\text { dimensionless velocity of the particle in the } \\
\text { simulation }\end{array}$ \\
\hline$V_{0}$ & $\begin{array}{l}\text { normalized settling velocity for particle in the } \\
\text { middle of the cross-section }\end{array}$ \\
\hline$V_{1}$ & $\begin{array}{l}\text { normalized settling velocity for particle close } \\
\text { to } 1 \text { wall }\end{array}$ \\
\hline$V_{2}$ & $\begin{array}{l}\text { normalized settling velocity for particle close } \\
\text { to } 2 \text { walls }\end{array}$ \\
\hline $\begin{array}{l}\Delta V= \\
\left(\max \left(V_{0}, V_{1}, V_{2}\right)\right. \\
- \\
\left.\min \left(V_{0}, V_{1}, V_{2}\right)\right)\end{array}$ & $\begin{array}{l}\text { deviation between the maximum and the } \\
\text { minimum velocities }\end{array}$ \\
\hline 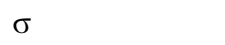 & surface tension \\
\hline$\mu$ & shear viscosity of the solutions \\
\hline & shear viscosity of the interfaces liquid-air. \\
\hline
\end{tabular}

\section{Acknowledgements}

We gratefully acknowledge financial support from Agence Nationale de la Recherche (ANR-05-JCJC-0234-01), E.S.A.
(MAP No. A099-108: C14914/02/NL/SH) and the French Space Agency (convention CNES/70980).

\section{References}

1 Hans-Joachim Schulze and Anh V. Nguyen, in Colloidal Science of Flotation, Surfactant Science Series 118, New York, 2004, ch. 1.

2 S. J. Neethling and J. J. Cilliers, Int. J. Miner. Process., 2003, 72, 267.

3 N. A. Bennani, A. Fujiwara, S. Takagi and Y. Matsumoto, Colloids Surf., $A, 2007,309,7$.

4 O. Pitois, C. Fritz, L. Pasol and M. Adler, Phys. Fluids, 2009, 21, 103304.

5 A. Saint-Jalmes, Y. Zhang and D. Langevin, Eur. Phys. J. E, 2004, 15, 53.

6 P. Stevenson, J. Colloid Interface Sci., 2005, 290, 603.

7 E. Lorenceau, N. Louvet, F. Rouyer and O. Pitois, Eur. Phys. J. E, 2009, 28, 293-304.

8 C. Monnereau and M. Vignes-Adler, J. Colloid Interface Sci., 1998, 202, 45.

9 W. Drenckhan, H. Ritacco, A. Saint-Jalmes and et al, Phys. Fluids, 2007, 19, 102101.

10 R. A. Leonard and R. Lemlich, AIChE J., 1965, 11, 18.

11 K. D. Danov, T. D. Gurkov, H. Raszillier and et al, Chem. Eng. Sci., 1998, 53, 3413.

12 A. J. Goldman, R. G. Cox and H. Brenner, Chem. Eng. Sci., 1967, 22, 637.

13 P. Ganatos, S. Weinbaum and R. Pfeffer, J. Fluid Mech., 1982, 124, 27.

14 A. Miyamura, S. Iwasaki and T. Ishii, Int. J. Multiphase Flow, 1981, 7, 41.

15 R. P. Chhabra, S. Agarwal and K. Chaudhary, Powder Technol., 2003, 129, 53.

16 R. P. Chhabra, M. Kumar and R. Prasad, Powder Technol., 2000, 113, 114.

17 M. E. Staben, K. P. Galvin and R. H. Davis, Chem. Eng. Sci., 2006, 61, 1932. 